\title{
An atypical form of AOA2 with myoclonus associated with mutations in SETX and AFG3L2
}

\author{
Cecilia Mancini ${ }^{1 \dagger}$, Laura Orsi ${ }^{2 \dagger}$, Yiran Guo ${ }^{3 \dagger}$, Jiankang $\mathrm{Li}^{4 \dagger}$, Yulan Chen ${ }^{4}$, Fengxiang Wang ${ }^{3}$, Lifeng Tian ${ }^{3}$, \\ Xuanzhu Liu', Jianguo Zhang ${ }^{4}$, Hui Jiang ${ }^{4,5,6}$, Bruce Shike Nmezi ${ }^{7}$, Takashi Tatsuta $^{8}$, Elisa Giorgio ${ }^{1}$, \\ Eleonora Di Gregorio ${ }^{9}$, Simona Cavalieri ${ }^{9}$, Elisa Pozzi ${ }^{1}$, Paolo Mortara ${ }^{2,10}$, Maria Marcella Caglio ${ }^{10,11}$, \\ Alessandro Balducci ${ }^{10,11}$, Lorenzo Pinessi 2,10, Thomas Langer ${ }^{8,12}$, Quasar S Padiath ${ }^{7}$, Hakon Hakonarson 3,13,14, \\ Xiuqing Zhang ${ }^{4,5,6}$ and Alfredo Brusco ${ }^{1,9^{*}}$
}

\begin{abstract}
Background: Hereditary ataxias are a heterogeneous group of neurodegenerative disorders, where exome sequencing may become an important diagnostic tool to solve clinically or genetically complex cases.

Methods: We describe an Italian family in which three sisters were affected by ataxia with postural/intentional myoclonus and involuntary movements at onset, which persisted during the disease. Oculomotor apraxia was absent. Clinical and genetic data did not allow us to exclude autosomal dominant or recessive inheritance and suggest a disease gene.
\end{abstract}

Results: Exome sequencing identified a homozygous c.6292C > T (p.Arg2098*) mutation in SETX and a heterozygous c.346G > A (p.Gly116Arg) mutation in AFG3L2 shared by all three affected individuals. A fourth sister (II.7) had subclinical myoclonic jerks at proximal upper limbs and perioral district, confirmed by electrophysiology, and carried the p.Gly116Arg change. Three siblings were healthy.

Pathogenicity prediction and a yeast-functional assay suggested p.Gly116Arg impaired m-AAA (ATPases associated with various cellular activities) complex function.

Conclusions: Exome sequencing is a powerful tool in identifying disease genes. We identified an atypical form of Ataxia with Oculoapraxia type 2 (AOA2) with myoclonus at onset associated with the c.6292C > T (p.Arg2098*) homozygous mutation. Because the same genotype was described in six cases from a Tunisian family with a typical $\mathrm{AOA} 2$ without myoclonus, we speculate this latter feature is associated with a second mutated gene, namely AFG3L2 (p.Gly116Arg variant).

We suggest that variant phenotypes may be due to the combined effect of different mutated genes associated to ataxia or related disorders, that will become more apparent as the costs of exome sequencing progressively will reduce, amplifying its diagnostics use, and meanwhile proposing significant challenges in the interpretation of the data.

Keywords: AFG3L2, Exome sequencing, Senataxin, SETX, Modifier genes, SCAR1, Ataxia with Oculomotor Apraxia Type 2, Autosomal recessive ataxia, Myoclonus

\footnotetext{
* Correspondence: alfredo.brusco@unito.it

${ }^{\dagger}$ Equal contributors

'Department of Medical Sciences, University of Torino, via Santena 19, 10126

Torino, Italy

${ }^{9}$ Medical Genetics Unit, Città della Salute e della Scienza University Hospital,

Torino 10126, Italy

Full list of author information is available at the end of the article
} 


\section{Background}

The hereditary ataxias are a highly genetically heterogeneous group of disorders phenotypically characterized by gait ataxia, incoordination of eye movements, speech, and hand movements, and usually associated with cerebellar atrophy. Autosomal dominant forms (Spinocerebellar ataxia, SCA) typically have adult-onset; conversely autosomal recessive ataxias (Spinocerebellar ataxias autosomal recessive, SCAR) usually have onset in childhood $[1,2]$.

Genetic tests needed to define the subtype are often long and laborious, due to the size of many ataxia genes (e.g., SYNE1, SACS, ATM, and SETX).

As a possible alternative to the gene-by-gene mutation screening, the application of Next Generation Sequencing technologies (NGS), involving simultaneous analysis of hundreds of selected exons or the entire sequencing of human coding genes (exome) are increasingly being used as a diagnostic tool, because of affordable costs and the availability of efficient bioinformatics tools. This approach becomes even more efficient in the case of a heterogeneous phenotype or unspecific clinical symptoms, which may make difficult a correct genetic diagnosis.

Beyond the mutation detection which is important to define a diagnosis for genetic counselling, future prenatal diagnosis and eventually therapy, exome analysis may also reveal unexpected findings, such as possible disease modifier genes [3].

In this study, we analyzed a single family with three sisters affected by ataxia with an atypical postural and intentional myoclonus. Exome sequencing revealed a homozygous SETX gene mutation, and an unexpected c.346G > A (p.Gly116Arg) variant in the AFG3L2 gene -responsible of SCA28- predicted to alter protein function. Clinical re-evaluation of the patients confirmed some typical feature of AOA2 and identified a fourth sibling carrying p.Gly116Arg with initial signs of myoclonus, suggesting variants in AFG3L2 may be also associated with myoclonus.

\section{Methods}

\section{Study subjects}

We identify three subjects from family ATA-2-TO (II.1, II.2, II.3 in Figure 1) presented with unsteadiness and difficulties in walking accompanied by a large spectrum of involuntary movements. Due to the early death of the mother and the unavailability of the father, we did not initially ascertain the type of transmission. As an initial workout, we excluded ataxias due to polyglutamine

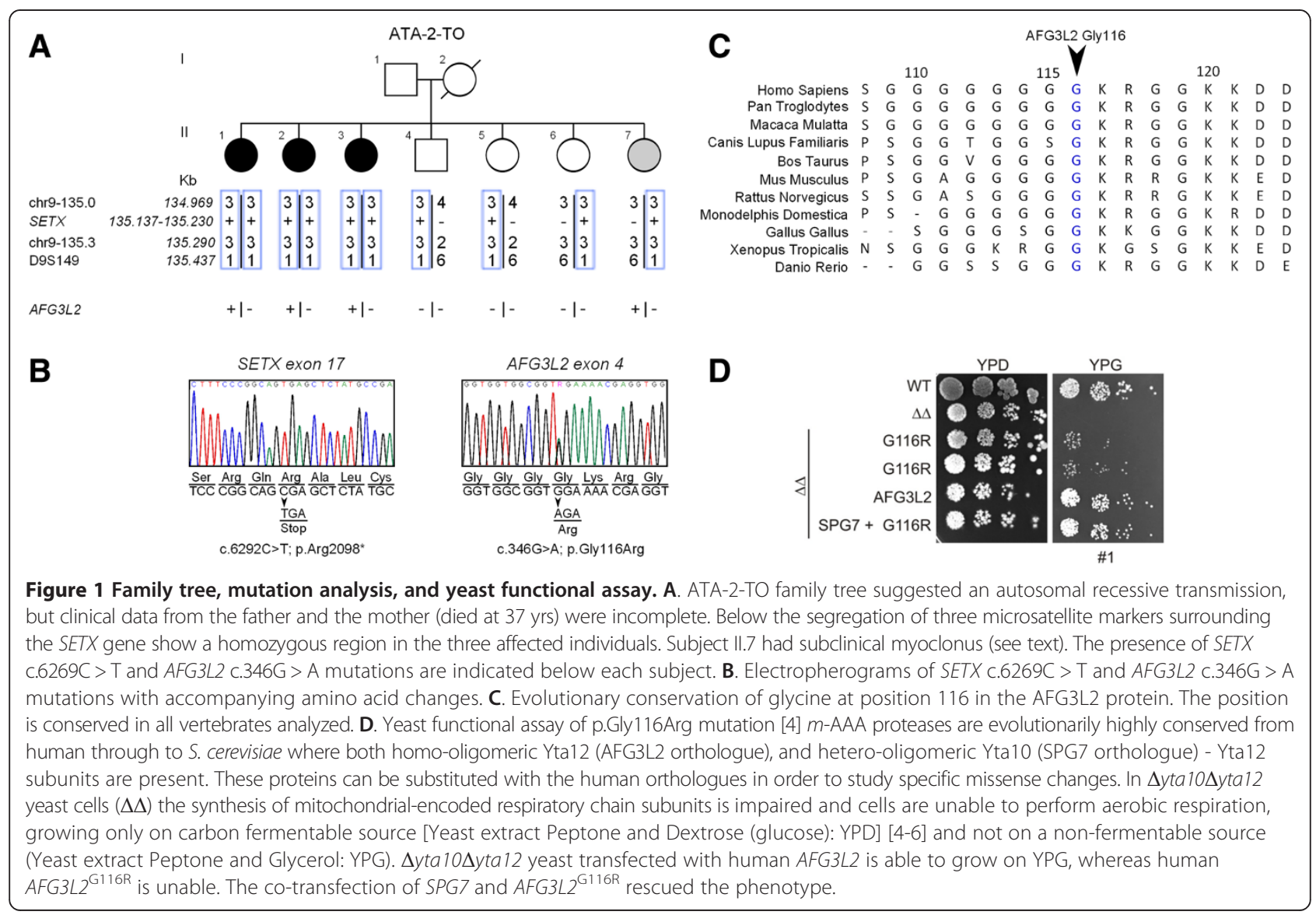


expansion (SCA1-3, 6, 7) and Friedreich ataxia; alphafetoprotein was available only at the end of the study. Short Tandem Repeats (STR) typing excluded SCA14 and SACS loci.

To evaluate the possible consanguinity in family ATA2-TO, after mutation discovery, we studied the following markers in the 9q34 region surrounding SETX: chr9_135.0 (5' -tgttatttgcactgggagga; 5' -agctcaatgtagctgttatttttct); chr 9_135.3 (5' -atgggatacatgcacacgttta; 5' -caatgcagctcacttgaaa actta); D19S149 (5'-gattgacctgtgaatttgtacagc; $5{ }^{\prime}$-tgttatgcc ttgctgttgct). Research was approved by the Internal Review Board of the Department of Medical Sciences, University of Torino. A written informed consent for publication was obtained from members participating in the study.

\section{Genetic analyses}

Genomic DNA was extracted from peripheral blood (Qiagen, Hilden, Germany) following the manufacturer instructions.

Exome capture was performed with Agilent SureSelect Human All Exon kit (Agilent Technologies, Santa Clara, CA, USA) according to the manufacturer protocols.

Sequencing was performed on an Illumina HiSeq 2000 machine using a standard pair-end read sequencing protocol (Illumina, San Diego, CA, USA) to generate sequencing reads of up to 90 cycles. We applied default settings in the Illumina pipeline to call bases from raw images, generating raw sequencing reads in the format of fastq files. We subsequently applied two independent analysis methods to perform alignment, variant calling and annotation. Pipeline 1 (P1) aligned fastq files to the human reference genome (UCSC hg19) with BWA then used Genome Analysis Tool Kit (version 1.4) to call variants, followed by utilizing Annovar and SnpEff for the variant functional annotation step. Pipeline 2 (P2) mapped fastq files to the human reference genome (UCSC hg19) with SOAP (version 2.21) and used SOAPsnp (version 1.05) for single nucleotide variant (SNV) detection, and used GATK for small insertion-deletion (indel) detection, followed by BGI self-developed programs to perform variant functional annotation.

We excluded variants that were: 1 ) out of exonic regions, 2) synonymous changes and 3) with minor allele frequency $(\mathrm{MAF})>0.5 \%$ in either the 1000 Genomes Project (http://www.1000genomes.org/), the NHLBI Exome Sequencing Project (ESP6500; http://evs.gs.washington.edu/EVS/), or our internal exome datasets. Variants near splicing donor/recipient sites and frameshift indels were given particular attention as they could cause pathogenic changes like exon-skipping or frameshifts. We filtered variants based on 1) evolutionary conservation, ie variants of PhyloP value $<0.95$ were considered to be in non-conserved regions thus discarded; 2) prediction of pathogenicity by PolyPhen and SIFT software.
Validation of the mutation was performed by Sanger sequencing in all members of the family with primers designed to amplify the exon 17 region of SETX (Reference sequence: NM_015046.5)(5'-tgggaactaattttccttctcattg and $5^{\prime}$-agcctggaagacagagcaagact).

\section{AFG3L2 mutation analysis and functional assay}

Clustal omega (http://www.clustal.org/omega/) was used for evolutionary conservation analysis. AFG3L2 (NM_006796) exon 4 was amplified and sequenced accordingly to previous published protocol [7]. For the yeast complementation assay a combination of human wild-type or mutant human AFG3L2 was expressed with or without wildtype paraplegin in a previously generated yeast strain Dyta10Dyta1.[5] AFG3L2 was mutagenized in the yeast expression constructs using the QuickChange XL SiteDirected Mutagenesis Kit (Stratagene, LaJolla, CA). Yeast cells were grown according to standard procedures at $30^{\circ} \mathrm{C}$ in YP medium containing $2 \%(\mathrm{w} / \mathrm{v})$ dextrose (glucose) (YPD). For testing the respiratory activity, yeast cells were grown at $30^{\circ} \mathrm{C}$ on solid state or in the liquid YP media containing 3\% (w/v) glycerol as the sole carbon source (YPG). Experiments were repeated three times with overlapping results.

\section{Results}

\section{Genetic analysis}

Whole exome sequencing on three family members (II.1, II.3, II.7) was performed with at least 57X coverage depth (Additional file 1: Table S1 and S2). Two analysis pipelines were used for each exome: P1 called $\sim 45,000$ SNVs and $\sim 7,500$ indels, P2 74,000 SNVs and $\sim 5,000$ indels. Further analysis was restricted to variants occurring in coding regions or at splicing sites, reducing SNVs to $\sim 17,000$ and indels to $\sim 700$ in P1, and $\sim 17,000$ SNVs and $\sim 630$ indels in P2 (Additional file 1: Table S1).

We initially analyzed the coding region of known ataxia causing genes assuming an autosomal recessive or dominant model of inheritance. We retained only rare (MAF $<0.5 \%$ in 1000 Genomes Project, the ESP6500 and CAG/BGI internal exome datasets) non-synonymous variants or splice site variants. A single homozygous $C$ to $\mathrm{T}$ variant on chr 9:135,163,655 causing a c.6292C $>\mathrm{T}$ (p.Arg2098*) in the SETX gene (NM_015046.5, OMIM "608465, http://www.omim.org/) was left after both P1 and P2 lists (Additional file 1: Table S3). This homozygous mutation was confirmed by Sanger sequencing in all affected individuals (Figure 1B), and haplotype analysis showed that it was derived from a common ancestor (Figure 1A). Although consanguinity in patients parents was denied, they were both born in a small village in Sicily increasing the likelihood they were consanguineous. The three sisters (II.1, II.2 and II.7) also shared the c.346G > A variant in the AFG3L2 gene 
changing amino acid glycine 116 into arginine (Additional file 1: Table S4). AFG3L2 encodes for a subunit of the hetero-oligomeric m-AAA protease (ATPases associated with various cellular activities), a component of the mitochondrial ATP-dependent metalloprotease located on the inner mitochondrial membrane, and its mutations in heterozygosis are associated with Spinocerebellar ataxia type 28 (SCA28) [8].

Segregation analysis showed that this variant was present also in the third affected sister II.3, but it was absent in II.4, II.5 and II.6 (Figure 1A). This substitution is not reported as validated polymorphism in Human variation resources (dbSNP(138), 1000 genomes, EVS, ExAC or HapMap databases), hit a highly conserved residue through all vertebrates (Figure 1C), it was predicted to be highly pathogenic by Mutation taster software (probability: 0.999; www.mutationtaster.org/) and to affect protein function with a score of 0.03 by SIFT Alignment (Median sequence conservation: 3.34; sift. jcvi.org/). The substitution is between the two mitochondrial transmembrane domains of AFG3L2.

We introduced the c.346G > A (p.Gly116Arg) mutation in the human full length $A F G 3 L 2$ cDNA. Expression in $\Delta y$ ta10 $\Delta y$ ta 12 cells did not restore mitochondrial respiratory activity as shown by the inhibition of growth on glycerol-containing media. Co-expression of SPG7 with AFG3L2 ${ }^{\text {G116R }}$ in $\Delta y$ ta10 $y$ ta12 cells substantially restored respiratory growth to normal levels (Figure 1D).

\section{Clinical findings}

Between 9 and 12 yrs, three subjects from family ATA-2TO (II.1, II.2, II.3 in Figure 1A) presented with unsteadiness and difficulties in walking accompanied by a large spectrum of involuntary movements such as myoclonic jerks at arms and hands, and/or choreic movements and facial dyskinesia. Myoclonic jerks were spontaneous and stimulus-insensitive, of small amplitude and arrhythmic. They were present at rest, on sustained posture and on action, involved proximal and distal segments of the upper limbs, and the perioral region. They attenuated over the course of the disease without disappearing.

Basal electroencephalography (EEG) and polygraphic EEG-EMG were normal and disease progression was slow. Patients developed truncal and limb ataxia with a progressive sensory-motor neuropathy that led them to difficulty walk without help after 5-8 years of disease. All patients were wheelchair-bound before $30 \mathrm{yrs}$.

Further signs included areflexia, distal absence of vibratory sensibility of the knees, and altered Somatosensory Evoked Potential.

Oculomotor abnormalities included nystagmus in all directions, hypometric saccades, saccadic pursuit, but no overt oculomotor apraxia. A mild limitation of the bilateral horizontal gaze was observed in II.3 (convergent strabismus) and a mild eyelid ptosis in II.1 was present after more than 30 years of disease.

One patient (II.3) showed a borderline intellectual disability (IQ = 77).

At last examination ( 40 yrs of disease duration, summary in Table 1), subjects presented with severe ataxia. Notably, all cases had an early menopause.

Skeletal abnormalities included a kyphoscoliosis (3/3) whereas pes cavus was not present.

Serum alpha-fetoprotein, obtained only at the last examination, was increased (Table 1).

Neuroradiology showed a marked cerebellar atrophy of hemispheres and vermis (Additional file 2: Figure S1).

Parents were reported healthy. The mother died at age 37 from pregnancy related complications, the father living at age 72 has been reported as unaffected, but he was unavailable for clinical examination.

\section{Patient II.7}

All siblings of the three affected individuals underwent a neurological investigation and did not show any neurologic phenotype except II.7. She reported occasional experience of imbalance, mainly after fatigue or stress at 36 yrs. At 42 yrs, neurological examination showed the presence of sporadic spontaneous myoclonus at proximal upper limbs and at the perioral district, confirmed by electromyography. Hyperreflexia at upper limbs, hyperactive patellar and ankle reflexes with a bilateral foot clonus and mild hypertonia at lower limbs with a weak Babinski sign, suggested a mild pyramidal involvement. No cerebellar ataxia, oculomotor abnormalities, and sensory deficits were present. MRI was not available.

\section{Discussion}

Diagnosis for rare inherited ataxias is hampered by the clinical overlap of heterogeneous disorders, and routine tests are often limited to repeat expansions, with rarer genes interrogated only in a research based setting. Targeted resequencing and exome sequencing are rapidly changing this approach demonstrating their utility as a diagnostic tool [9].

Ataxia and sensory-motor neuropathy are typical symptoms of AOA2 that may be complicated by additional movement disorders during the disease course. Chorea/choreoathetosis, facial dyskinesia, dystonia, head/ postural tremor are described in $14 \%$ of AOA2 patients, but they were never reported at onset [10-12]. Myoclonus is described in only one AOA2 case [13].

We studied an atypical form of AOA2 with involuntary movements/myoclonus at onset shared by three sisters. None had oculomotor apraxia (OMA), reported in half of the AOA2 cases. However, all had hypometric saccades with a staircase pattern, a sign recently suggested as more reliable for OMA [14]. 
Table 1 Neurological and biochemical features of AOA2 patients

\begin{tabular}{l} 
Patients \\
\hline Sex/age \\
Age at onset of gait ataxia \\
Disease duration (years) \\
Interval onset-wheelchair \\
Initial symptom \\
Oculomotor anomalies \\
OMA \\
Hypometric saccades \\
Saccadic pursuit \\
Strabismus \\
Ptosis
\end{tabular}

Pyramidal signs

Plantar Reflex

\section{Involuntary movements}

Head/hand tremor

Myoclonus

Dystonia

Facial Dyskinesia/ Choreic movements

Other Extra-pyramidal signs

\section{Reflexa}

\section{Peripheral neuropathy}

Distal amiotrophy (UL, LL)

Deep sensory loss

Sensory loss

Pain and light touch

Sensory motor neuropathy

\section{Others}

SARA Score

IQ (WAISS)

Progression of disability

Pes cavus

Kyphoscoliosis

Early menopause (yrs)

\section{Brain MRI}

Vermian Atrophy

Brainstem atrophy

\section{Biochemistry}

AFP, ng/ml (normal level $<7$ ng/ml)

Cholesterol

\section{Genetics}

SETX homozygous mutation

AFG3L2 heterozygous mutation

Il.1
F/51
12
39
21
Chorea/myoc
-
+++
$+(33$ yrs.)
-
$+(39$ yrs $)$

$++(24$ yrs.)

UL, LL

$+(33 \mathrm{yrs}$ )

$+$

Bradikynesia/hypomimia

Absent UL/LL

$+++$

$+++$

$+++$

DecreasedUL;absent LL

$+++$

$27 / 40$

$93(102 / 83)$

$17 ; 21 ; 36^{* *}$

-

+++

33

$+++$

-

19

norm
$+++$

$+(34 \mathrm{yrs})^{*}$

$-$

$-$

Extension

$++(30$ yrs.)

UL, LL

$+(40$ yrs.)

$+$

Bradikynesia/hypomimia

Absent UL/LL

$+++$

$+++$

$-$

Decreased UL;absent LL

$+++$

$27 / 40$

$87(86 / 89)$

$17 ; 21 ; 37^{* *}$

-

$+++$

32

$+++$

$-$

61

norm

c.6292C > T (p.Arg2098*)

c.346G > A (p.Gly116Arg)

\section{3}

F/44

12

32

23

Chorea/myoclonia

$+++$

$+(26 \text { yrs. })^{*}$

$++(32$ yrs.)

UL, LL

Bradikynesia/hypomimia Absent UL/LL

$+++$

$+++$

$-$

Decreased UL; loss LL

$+++$

$23 / 40$

$77(78 / 78)$

$17 ; 21 ; 38^{* *}$

$+++$

34

$+++$

$++$

37

norm

c.6292C > T (p.Arg2098*) c.346G > A (p.Gly116Arg)

Legend: - None; + Mild; ++ Moderate; +++ Severe; norm: normal; na: not available; *disease duration; UL Upper Limbs; LL Lower Limbs.

**Progression of disability indicates gait possible with one help; with a double help; wheelchair bounded. WAISS Weschler adult intelligence Scale Score; total IQ, and verbal/non verbal IQ (among brackets) are indicated. 
A mild limitation of the lateral gaze and a mild convergent strabismus in the left eye occurred in II-3 after 26 yrs. of disease (12-38\% of AOA2 patients) [10,11]. Ptosis, never reported in AOA2, may be a late sign of disease, and appeared unilaterally in the eldest patient at 51 yrs. Further uncommon features included a mild cognitive impairment in II.3 [11]. Skeletal abnormalities included a kyphoscoliosis (3/3) never reported in AOA2, whereas pes cavus, a finding present in almost all reported AOA2 cases was not present $[10,11]$.

We noted that all three sisters presented a premature menopause between 32 and 34 yrs, a feature already occasionally reported in AOA2 $[15,16]$. This may suggest an association between SETX mutations and premature ovarian failure/premature menopause that deserves further studies.

Among the other four siblings, II.7 showed spontaneous mild myoclonus and hyperreflexia at upper and lower limbs.

Exome sequencing identified a homozygous c.6292C $>\mathrm{T}$ (p.Arg2098*) mutation in this gene. This same mutation in homozygosis had already been described in six AOA2 Tunisian patients (Family E) [17], who showed a typical form of disease without involuntary movements/myoclonus. This finding suggested that a second genetic determinant may have combined its effect with the SETX mutation to give the atypical phenotype in our family. Exome data also revealed the c.346G > A (p.Gly116Arg) missense change in $A F G 3 L 2$, encoding for a mitochondrial protease. In the inner membrane of human mitochondria two different functionally active $m$-AAA isoenzymes are present: a homo-oligomeric AFG3L2 complex and a hetero-oligomeric complex where AFG3L2 subunits assemble with paraplegin. Mutations in this gene cause both an autosomal dominant spinocerebellar ataxia (SCA28) characterized by ophtalmoplegia/ptosis and oculomotor alterations $[7,8]$ or an autosomal recessive form of early onset spastic ataxia with neuropathy and myoclonic epilepsy (SPAX5) [18].

Yeast experiments demonstrated that only homooligomeric $m$-AAA protease complexes composed by AFG3L2 ${ }^{\text {G116R }}$ subunits are functionally impaired. We concluded that AFG3L2 ${ }^{\mathrm{G} 116 \mathrm{R}}$ is likely pathogenic, but behaves differently from AFG3L2 mutations causing SCA28, that fail to be rescued by paraplegin co-expression [8]. AFG3L2 mutations reported in the literature mainly cluster in the protease C-terminal domain $[7,8,19]$. Localization of p.Gly116Arg at the N-terminal portion of the protein, suggests a different effect on the protein that may lead to a so far never reported phenotype.

Indeed, p.Gly116Arg was present in the three affected sisters and II.7 only. The latter had subclinical myoclonus at age 41. All four siblings without AOA2 (including II.7) were carriers of the c.6292C > T mutation in SETX.

\section{Conclusion}

Using exome sequencing, we identified an atypical form of AOA2 with myoclonus at onset associated with the c.6292C > T (p.Arg2098*) homozygous mutation. We hypothesize that the combination of AOA2 with the c.346G > A (p.Gly116Arg) mutation in $A F G 3 L 2$, is responsible for the variant phenotype seen in three patients. The p.Gly116Arg variant in AFG3L2 may be a candidate for myoclonus, but we cannot exclude this is an initial symptom that will evolve into a more complex phenotype.

We suggest that variant phenotypes may be due to the combined effect of different mutated genes associated to ataxia or related disorders, that will become more apparent as the costs of exome sequencing progressively will reduce, amplifying its diagnostics use, and meanwhile proposing significant challenges in the interpretation of the data.

\section{Additional files}

Additional file 1: Table S1. Exome sequencing summary statistics. Table S2. Rare variants candidate list from two independent analysis pipelines base on autosomal recessive model of inheritance and multiple steps of filtering. Table S3. Homozygous variant shared by both patients before filtering on allele frequency and variant annotation. Table S4. Heterozygous variant shared by both patients before filtering on allele frequency and variant annotation.

Additional file 2: Figure S1. MRI of $A O A 2$ patients. Coronal ( $A$ and $B$ ) and sagittal ( $C$ and D) T1-weighted brain resonance magnetic imaging slices. Panel A and B represent patient II.1 at 41 yrs (29 yrs of disease duration), and panels $C$ and D represent II.3 at 34 yrs (22 yrs of disease duration). A marked cerebellar hemispheric and vermian atrophy was present in both patients (arrow).

\section{Competing interests}

Jiankang Li, Xuanzhu Liu, Yulan Chen, Jianguo Zhang, Xiuqing Zhang, and Hui Jiang are employees of BGl-Shenzhen. No further financial disclosures.

\section{Authors' contributions}

Research project: CM, ABr. Organization: CM, YG, JL,TL, QSP, XZ, ABr. Execution: CM, LO, YG, XL, JL, HJ, FW, LT, JZ, YC, BSN, TT, EDG, EG, SC, EP, PM, $M M C, A B, L P, H H$. Writing of the first draft: $C M, L O, T L, Q S P, A B r$. Review and Critique: CM, LO, YG, JL, YC, FW, LT, JZ, HJ, BSN, TT, EDG, EG, SC, EP, PM, $M M C, A B, L P, T L, Q S P, H H, X Z, A B r$. All authors read and approved the final manuscript.

\section{Acknowledgments}

We are grateful to the family members who participated in this study.

\section{Funding}

This work was funded by Telethon Research grant GGP12217, Associazione E. E. Rulfo, PRIN 2010_2011 (Grant 20108WT59Y) (to A. Brusco), Research Grant from the Shenzhen Municipal Government of China (NO.

CXZZ20130517144604091), the Shenzhen Key Laboratory of Genomics (NO.CXB200903110066A) and the Guangdong Enterprise Key Laboratory of Human Disease Genomics (NO.2011A060906007), University of Pittsburgh to Q. S. Padiath, and Institutional Development Funds to H. Hakonarson at the Center for Applied Genomics of The Children's Hospital of Philadelphia.

\section{Web resources}

1000 genomes, http://www.1000genomes.org/ dbSNP(138), http://www.ncbi.nlm.nih.gov/snp/ 
ExAC, Exome Aggregation Consortium, Cambridge, MA (URL: http://exac. broadinstitute.org) [January, 2015 accessed]

EVS, http://evs.gs.washington.edu/

HapMap project, http://www.hapmap.org

MutationTaster, http://www.mutationtaster.org/

Online Mendelian Inheritance in Man (OMIM), http://www.OMIM.org

PolyPhen-2, http://www.genetics.bwh.harvard.edu/pph2/

SIFT, http://sift.bii.a-star.edu.sg/

\section{Author details}

'Department of Medical Sciences, University of Torino, via Santena 19, 10126 Torino, Italy. ${ }^{2}$ Struttura Complessa Neurologia I, Department of Neuroscience and Mental Health, Città della Salute e della Scienza University Hospital, Torino 10126, Italy. ${ }^{3}$ Center for Applied Genomics, The Children's Hospital of Philadelphia, Philadelphia, PA 19104, USA. BGI-Shenzhen, Shenzhen 510803, China. ${ }^{5}$ Shenzhen Key Laboratory of Genomics, Shenzhen 518083, China. ${ }^{6}$ The Guangdong Enterprise Key Laboratory of Human Disease Genomics, BGl-Shenzhen, Shenzhen 510803, China. 'Department of Human Genetics, Graduate School of Public Health, University of Pittsburgh, Pittsburgh, PA 15261, USA. ${ }^{8}$ Institute for Genetics, Center for Molecular Medicine (CMMC), Cologne Excellence Cluster on Cellular Stress Responses in Aging-Associated Diseases (CECAD), University of Cologne, Cologne 50931, Germany. ${ }^{9}$ Medical Genetics Unit, Città della Salute e della Scienza University Hospital, Torino 10126, Italy. ${ }^{10}$ Department of Neuroscience, University of Torino, Torino 10126, Italy. ${ }^{11}$ Division of Neurology III, Department of Neuroscience and Mental Health, Città della Salute e della Scienza University Hospital, Torino 10126, Italy. ${ }^{12}$ Max-Planck-Institute for Biology of Aging, Cologne 50931, Germany. ${ }^{13}$ Division of Human Genetics, The Children's Hospital of Philadelphia, Philadelphia, PA 19104, USA. ${ }^{14}$ Department of Pediatrics, The Perelman School of Medicine, University of Pennsylvania, Philadelphia, PA 19104, USA.

Received: 19 November 2014 Accepted: 26 February 2015

Published online: 19 March 2015

\section{References}

1. Jayadev S, Bird TD. Hereditary ataxias: overview. Genetics Med. 2013;15(9):673-83.

2. Anheim M, Tranchant C, Koenig M. The autosomal recessive cerebellar ataxias. N Engl J Med. 2012;366(7):636-46.

3. Craigen WJ, Graham BH, Wong LJ, Scaglia F, Lewis RA, Bonnen PE. Exome sequencing of a patient with suspected mitochondrial disease reveals a likely multigenic etiology. BMC Med Genet. 2013;14:83.

4. Bonn F, Pantakani K, Shoukier M, Langer T, Mannan AU. Functional evaluation of paraplegin mutations by a yeast complementation assay. Hum Mutat. 2010;31(5):617-21.

5. Koppen M, Langer T. Protein degradation within mitochondria: versatile activities of AAA proteases and other peptidases. Crit Rev Biochem Mol Biol. 2007;42(3):221-42.

6. Nolden M, Ehses S, Koppen M, Bernacchia A, Rugarli El, Langer T. The m-AAA protease defective in hereditary spastic paraplegia controls ribosome assembly in mitochondria. Cell. 2005;123(2):277-89.

7. Cagnoli C, Stevanin G, Brussino A, Barberis M, Mancini C, Margolis RL, et al. Missense mutations in the AFG3L2 proteolytic domain account for approximately $1.5 \%$ of European autosomal dominant cerebellar ataxias. Hum Mutat. 2010;31(10):1117-24.

8. Di Bella D, Lazzaro F, Brusco A, Plumari M, Battaglia G, Pastore A, et al. Mutations in the mitochondrial protease gene AFG3L2 cause dominant hereditary ataxia SCA28. Nat Genet. 2010;42(4):313-21.

9. Nemeth AH, Kwasniewska AC, Lise S, Parolin Schnekenberg R, Becker EB, Bera KD, et al. Next generation sequencing for molecular diagnosis of neurological disorders using ataxias as a model. Brain. 2013;136(Pt 10):3106-18.

10. Nanetti L, Cavalieri S, Pensato V, Erbetta A, Pareyson D, Panzeri M, et al. SETX mutations are a frequent genetic cause of juvenile and adult onset cerebellar ataxia with neuropathy and elevated serum alpha-fetoprotein. Orphanet J Rare Dis. 2013:8:123.

11. Anheim M, Monga B, Fleury M, Charles P, Barbot C, Salih M, et al. Ataxia with oculomotor apraxia type 2: clinical, biological and genotype/phenotype correlation study of a cohort of 90 patients. Brain. 2009;132(Pt 10):2688-98.
12. Tazir M, Ali-Pacha L, M'Zahem A, Delaunoy JP, Fritsch M, Nouioua S, et al. Ataxia with oculomotor apraxia type 2: a clinical and genetic study of 19 patients. J Neurol Sci. 2009;278(1-2):77-81.

13. Le Ber I, Bouslam N, Rivaud-Pechoux S, Guimaraes J, Benomar A, Chamayou C, et al. Frequency and phenotypic spectrum of ataxia with oculomotor apraxia 2: a clinical and genetic study in 18 patients. Brain. 2004;127(Pt 4):759-67.

14. Panouilleres M, Frismand S, Sillan O, Urquizar C, Vighetto A, Pelisson D, et al. Saccades and eye-head coordination in ataxia with oculomotor apraxia type 2. Cerebellum. 2013;12(4):557-67.

15. Lynch DR, Braastad CD, Nagan N. Ovarian failure in ataxia with oculomotor apraxia type 2. Am J Med Genet A. 2007;143A(15):1775-7.

16. Gazulla J, Benavente I, Lopez-Fraile IP, Modrego P, Koenig M. Sensorimotor neuronopathy in ataxia with oculomotor apraxia type 2. Muscle Nerve. 2009;40(3):481-5.

17. Hammer MB, El Euch-Fayache G, Nehdi H, Saidi D, Nasri A, Nabli F, et al. Clinical and molecular findings of ataxia with oculomotor apraxia type 2 (AOA2) in 5 Tunisian families. Diagn Mol Patho. 2012;21(4):241-5.

18. Pierson TM, Adams D, Bonn F, Martinelli P, Cherukuri PF, Mullikin For The Nisc Comparative Sequencing Program JC, et al. Whole-exome sequencing identifies homozygous AFG3L2 mutations in a spastic ataxia-neuropathy syndrome linked to mitochondrial m-AAA proteases. PLoS Genet. 2011;7(10):e1002325

19. Edener U, Wollner J, Hehr U, Kohl Z, Schilling S, Kreuz F, et al. Early onset and slow progression of SCA28, a rare dominant ataxia in a large four-generation family with a novel AFG3L2 mutation. Eur J Hum Genet. 2010;18(8):965-8.

\section{Submit your next manuscript to BioMed Central and take full advantage of:}

- Convenient online submission

- Thorough peer review

- No space constraints or color figure charges

- Immediate publication on acceptance

- Inclusion in PubMed, CAS, Scopus and Google Scholar

- Research which is freely available for redistribution 